\title{
Use of abbreviated mental status examination in the initial assessment of overdose patients
}

\author{
K. S. MERIGIAN, ${ }^{1}$ J. R. HEDGES, ${ }^{1}$ J. R. ROBERTS, ${ }^{1}$ \\ R. A. CHILDRESS, ${ }^{1}$ M. A. NIEHAUS ${ }^{2} \&$ N. FRANKLIN ${ }^{2}$ \\ ${ }^{1}$ Department of Emergency Medicine, University of Cincinnati College of Medicine, and \\ ${ }^{2}$ University of Cincinnati Medical Center, Emergency Nursing, Center for Emergency \\ Care, Cincinnati, USA
}

\section{SUMMARY}

Application of formal mental status testing in the emergency department (ED) to assess cognitive function has been hampered by the lack of a rapidly applied instrument. An Abbreviated Mental Status Examination (AMSE) with 10 test items that can be administered within five minutes by nursing personnel is described. Evaluation of the instrument on 296 ambulatory ED patients with grossly normal neurologic function showed that $93 \%$ of patients had a total score of seven or more correct answers and $83 \%$ of patients had eight or more correct answers.

Application of the AMSE to 375 acute drug overdose patients at the same hospital showed a significant correlation with Glasgow Coma Scale (GCS) score. An AMSE score of seven or less was found to be more sensitive than a GCS score of 13 or less for admission to the intensive care unit (ICU) and complications in the ICU $(P<0.001)$. The AMSE score may serve as a useful tool for stratifying cognitive function in acute drug overdose patients and for identifying patients at increased risk for an adverse outcome from their overdose.

\section{INTRODUCTION}

Evaluation of the cognitive function of patients in the emergency department (ED) has generally been limited by the time required for performance of cognitive function tests. The most popular tests used by psychiatrists include the Kahn's Mental Status

Correspondence: Dr ferris R. Hedges, Department of Emergency Medicine, University of Cincinnati College of Medicine, 234 Goodman Street, Cincinnati, OH 45267-0769, USA. 
Questionnaire (Kahn et al., 1960), the Mini-Mental State Examination by Folstein (Folstein et al., 1975), the Short Portable Mental Status Questionnaire by Pfeiffer (Pfeiffer, 1975), the Cognitive Capacity Screening Examination by Jacobs (Jacobs et al., 1977), and the Mattis Dementia Rating Scale (Mattis, 1976). These tests require a $\stackrel{\rho}{\circ}$ minimum of five and often up to 15 minutes to administer in alert co-operative patients (Nelson et al., 1986). Some skill on the part of the examiner and considerable persistence on the part of the patient are required. Hence these empiric mental status examinations are seldom used in the emergency department.

Zun \& Gold (1986) recently surveyed emergency physicians to determine the form and content of their mental status examination. They found that $19 \%$ of American Board of Emergency Medicine certified physicians never performed a mental status $\vec{\omega}$ examination while another $20 \%$ used it 'occasionally.' Nearly all respondents $(97 \%)$ stated they would use a short standardised mental status examination if one were shown useful in the ED. Zun \& Gold also noted that no currently proposed mental status examination has been evaluated for use in the ED.

Because cognitive impairment may reflect significant underlying metabolic dysfunction of the cerebrum, we evaluated an Abbreviated Mental Status Examination (AMSE) for its ability to denote normal cognitive function in ambulatory patients presenting to the ED and its ability to identify reduced cognitive function in patients presenting with significant drug intoxication. A test which can identify significant cognitive dysfunction prior to the development of a comatose state should be beneficial in evaluation and monitoring of drug-induced metabolic cerebral dysfunction in the ED. We specifically addressed the question of whether the AMSE could serve as $\frac{0}{9} \infty$ early prognosticator of serious metabolic dysfunction in overdose patients.

\section{METHODS}

\section{AMSE Development and Overview}

Initially, we used a 30 question menu by Dick et al. (1984) to assess mental status in our urban teaching hospital ED population. Although the menu of Dick et al. is more easily administered than other mental status examinations, our patients were unable to complete the examination either because of illiteracy, a decreased attention span, or emotional instability. On reviewing the initial menu of questions, 10 inquiries or commands were consistently answered or performed correctly by patients who exhi- $\bigcirc$ bited alert and oriented behaviour when admitted to the ED without an obvious altered mental status. These 10 inquiries or commands comprise the AMSE we evaluated (Table 1).

Evaluation of the AMSE proceeded in two steps. First, determination of the normal range of values was obtained in ambulatory patients presenting to our ED. Second, the AMSE was evaluated on patients with acute drug ingestion and correlated with the Glasgow Coma Scale (GCS) score and subsequent patient outcome measures. Although oc the GCS was originally devised for prognostication of head injured patients (Teasdale $\&$ Jennett, 1974), it is commonly applied to other populations with cognitive impairment and hence represents an appropriate tool for comparison. 
Table 1 Abbreviated Mental Status Examination

What time is it?

(To within 2 hours of exact time)

What day is it?

(Correct day of week)

What month is it?

(Correct month)

What city are we in?

(Correct city)

What is $25-7=$ ?(18)

What is $10-2=?(8)$

Identify a PEN

Identify WATCH

Follow three stage commands:

Right index finger to tip of nose

Left index finger to right ear
Incorrect

[]

\section{Evaluation of AMSE in Ambulatory Non-Overdosed Patients}

Ambulatory patients over 14 years of age presenting to an urban teaching hospital ED were included in this study if the nurse performing patient triage assessed the patient as having grossly normal neurologic function. Patients with severe acute medical disorders, such as acute chest pain or congestive heart failure were excluded from this population (one each excluded). Patients who were unco-operative (one excluded) or had a history of recent alcohol or sedative ingestion were also excluded (seven excluded).

The triage nurses were instructed in the use of the AMSE score by one of the investigators (K.S.M., M.A.N. or N.F.) following a protocol approved by the Institutional Review Board. All AMSE testing was completed within five minutes.

Patients were subsequently categorised by presenting complaint and total scores were compared after grouping of patients with and without acute or chronic pain, with or without trauma, and with or without an underlying psychiatric disorder (e.g., major depression or schizophrenia). Group score rank orders were compared using the Kruskal-Wallis test. The 10 components of the AMSE score were assessed individually to determine the error rate for each component and to determine if there was a significant error rate difference amongst the components. Error rates were compared using a Chi-Square analysis. Stat Pak statistical software (Northwest Analytical Co., Portland, OR) was used with a significance level of $P<0.05$ throughout.

\section{Evaluation in Overdose Population}

Patients presenting to an urban teaching hospital ED with an acute drug overdose were assessed by measurement of a presenting AMSE score and GCS score in the ED. No patients were excluded for unco-operativeness during this portion of the study. When a patient would not answer a question, the response was considered incorrect. Correlation of test scores was performed by linear regression analysis and statistical significance 
Table 2 Complications of overdose patients

\begin{tabular}{lc}
\hline Complications documented in the ICU & No. occurrences* \\
\hline Aspiration pneumonia & 5 \\
Hypoventilation/hypoxia & 4 \\
Respiratory arrest & 3 \\
Pulmonary oedema & 3 \\
Pneumothorax & 1 \\
Seizure & 9 \\
Hypotension requiring treatment & 5 \\
Dysrhythmia requiring treatment & 7 \\
Acute renal failure & 3 \\
Gastrointestinal bleeding & 3 \\
Ileus & 2 \\
Rhabdomyolysis & 2 \\
\hline
\end{tabular}

*Some patients had multiple complications.

determined by the Pearson Moment Coefficient. Furthermore, the patient's GCS scoref on presentation and AMSE score were compared with outcome measures including th need for admission to the intensive care unit (ICU), intubation in the ED or ICU, an $\vec{f}$ complications in the ICU (Table 2). The AMSE score and the GCS score wer\& compared for sensitivity of prediction of outcome criteria using the binomial distribux tion for $95 \%$ confidence intervals and McNemar's test (Kramer \& Feinstein, 1981)

\section{RESULTS}

\section{Determination of Normal Range}

Two hundred and ninety six patients were scored to determine the normal range of the AMSE score. These patients had a mean ( \pm standard deviation) total AMSE score of $8 \cdot 80 \pm 1 \cdot 50$. The histogram for the frequency of total AMSE scores is shown (Fig. 1). score of less than seven was seen in $7 \%$ of the total population, whereas, a score of les than eight was seen in $17 \%$ of the population. Although the triage nurses were instructed to apply the test only to patients they considered neurologically intact, 16 of the 28 patients with a total AMSE score less than seven and 26 of the 56 patients with $\frac{O}{2}$ total score less than eight were disorientated to either time, place, or date. Of the test items, 'subtraction by seven' had the most incorrect answers (43\% of the time incorrect). The multiple stage commands and 'subtraction by two' were also misse $\mathbb{9}$ significantly more frequently (incorrect $15-20 \%$ of the time) than the remainder of the्్ test items.

Comparison of the groups with or without acute or chronic pain and trauma disclose no significant differences in group score rank order (Table 3). Division of the patient population into groups with and without a psychiatric presenting complaint, however 


\section{AMBULATORY PATIENTS}

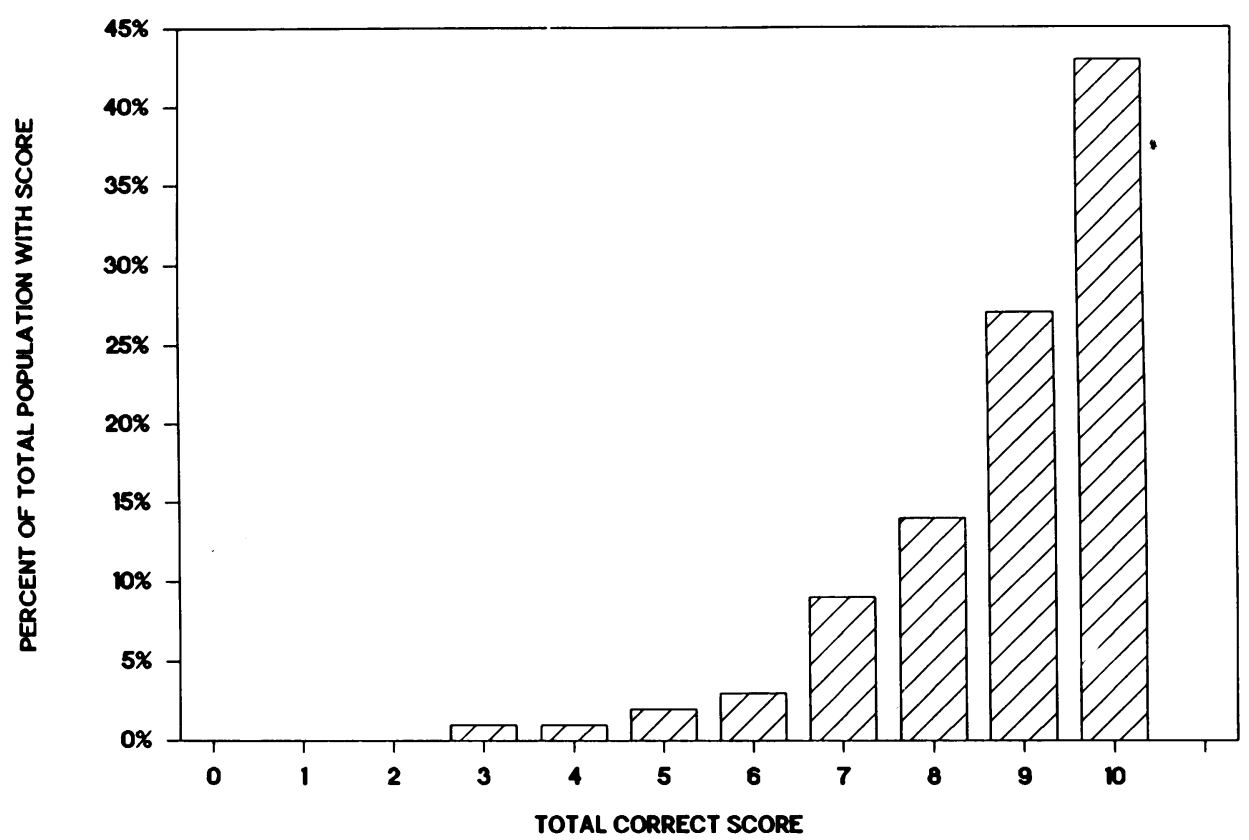

Fig. 1. Distribution of ambulatory emergency department patients with total Abbreviated Mental Status Examination scores.

Table 3 Total scores as a function of grouping

\begin{tabular}{lccccc}
\hline Feature & $*$ With feature & $($ No. $)$ & $*$ Without feature & $($ No. $)$ & $P$ value \\
\hline Acute pain & $8.77 \pm 1.44$ & $(101)$ & $8.81 \pm 1.55$ & $(195)$ & NS \\
Chronic pain & $8.80 \pm 1.93$ & $(10)$ & $8.80 \pm 1.49$ & $(286)$ & NS \\
Trauma & $9.08 \pm 1.20$ & $(36)$ & $8.76 \pm 1.54$ & $(260)$ & NS \\
Major psychiatric disorder & $7.58 \pm 2.30$ & $(26)$ & $8.91 \pm 1.35$ & $(270)$ & $<0.003$ \\
\hline
\end{tabular}

*All values shown as mean \pm standard deviation.

was significant $(P<0.005)$. Of those without a psychiatric diagnosis, the mean score was $8.91 \pm 1 \cdot 35$. Of the patients with a psychiatric complaint, the mean score was $7 \cdot 58 \pm 2 \cdot 30$.

\section{Correlation with Outcome in Overdose Patients}

A total of 375 overdose patients were assessed for a correlation of their AMSE and the GCS score. The patient population had a mean age of 29.8 years and was $43 \%$ male and 
Table 4 Correlation of score performance with outcome in overdose patients

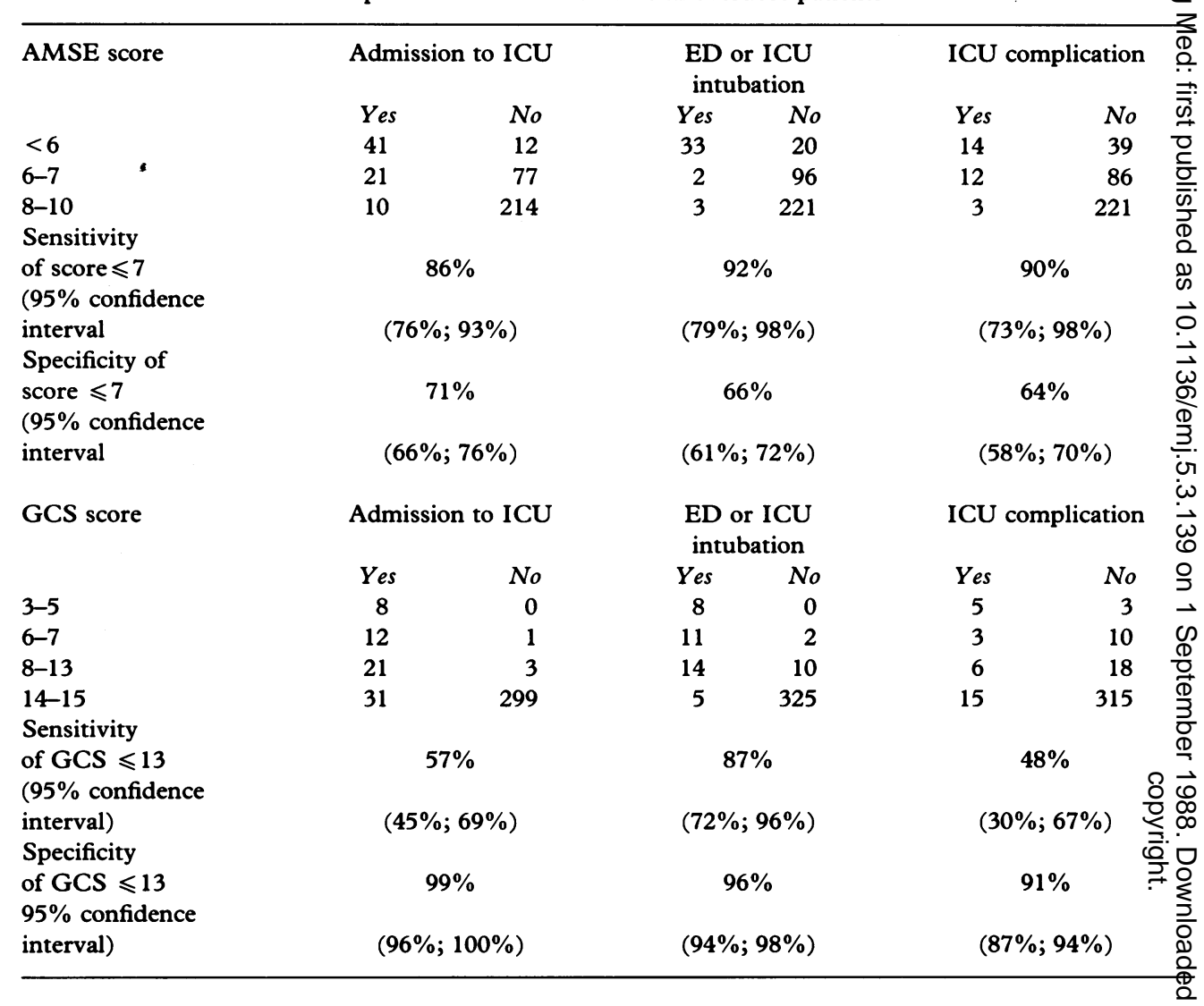

$72 \%$ white. The linear correlation was: AMSE $=-5.49+0.90 \times \mathrm{GCS} ; \mathrm{R}^{2}=0.5$ $(P<0.001)$. Both the AMSE and the GCS scores correlated with the admission of patients to the ICU, patient intubation in the ED or ICU, and the presence of complications in the ICU (Table 4). An AMSE score of seven or less was more sensitive for ICU admission and ICU complications than a GCS score of 13 or less $(P<0.001)$

\section{DISCUSSION}

We have shown that the total AMSE score in an urban population of ambulator patients presenting to the emergency department (who appear neurologically intact) is 'seven or greater' $93 \%$ of the time and 'eight or greater' $83 \%$ of the time. If patient with disorientation are excluded, a score of 'seven or greater' was seen in over $96 \%$ of patients and a score of 'eight or greater' was found in over $89 \%$ of patients. The cutoff less useful for patients with psychiatric complaints (such as major depressive disorde and schizophrenia). Errors in arithmetic may reflect the educational level of the urbain 
population assessed. However, a score of 'eight or greater' denotes acceptable cognitive function in our urban teaching hospital population.

Although the AMSE score is expected to be more sensitive for cognitive impairment than the GCS score, strong correlation between the GCS score and the AMSE score was anticipated and found in our patients with acute drug ingestion. In addition, we found a strong association with admistion to the ICU, intubation in the ED or ICU, and the presence of complications in the ICU. Hence, an AMSE score of 'seven or less' appears to represent a sensitive marker for significant drug-induced cognitive impairment. Although a low AMSE is not specific for acute drug intoxication (as seen in our population of ambulatory patients), when applied to the acute drug overdose population, the AMSE score does appear to be a sensitive prognosticator of adverse outcome.

We conclude that the AMSE is an empiric test rapidly performed which can be used to semiquantitatively denote cognitive impairment. Overdose patients with an AMSE score of 'seven or less' appear to be at greater risk for complications of their overdose than patients presenting with an AMSE score of 'eight or greater.' While we do not advocate that the AMSE be used as a criterion upon which to base treatment or disposition, the AMSE does appear to be a helpful prognosticator. Patients with a low AMSE should be monitored closely during their ED evaluation and therapy. The AMSE may prove to be a useful tool for objectively stratifying overdose patients with cognitive impairment. Further evaluation of the AMSE for screening other ED patient populations with potential for cognitive impairment appears warranted.

\section{REFERENCES}

Dick J.P. R., Guiloff R. J. \& Stewart A. (1984) Mini-mental state examination in neurological patients. fournal of Neurology Neurosurgery \& Psychiatry 47, 496-9.

Folstein M. F., Folstein S. E. \& McHugh P. R. (1975) Mini-mental state. fournal of Psychiatric Research 12, 89-198.

Jacobs J. W., Bernard M. R., Belgado A. \& Strain J. J. (1977) Screening for organic mental syndromas in the medically ill. Annals of Internal Medicine 86, 40-6.

Kahn R. L., Goldfarb A. I., Polack M. \& Peck A. (1960) Brief objective measures for the determination of mental status in the aged. American fournal of Psychiatry 117, 326-8.

Kramer M.S. \& Feinstein A.R. (1981) Clinical biostatistics. The biostatistics of concordance. Clinical Pharmacology and Therapeutics 299, 111-23.

Mattis S. (1976) Mental status examination for organic mental syndromes in the elderly patient. In Geriatric Psychiatry, Bellak L. \& Karasu T. E. (eds), Grune \& Stratton, New York.

Nelson A., Fogel B. S. \& Faust D. (1986) Bedside cognitive screening instruments: a critical assessment. fournal of Nervous and Mental Disease 174, 73-83.

Pfeiffer E. (1975) A short portable mental status questionnaire for the assessment of organic brain deficit in elderly patients. Fournal of the American Geriatric Society 23, 433-41.

Teasdale G. \& Jennett B. (1974) Assessment of coma and impaired consciousness. Lancet 2, 81-4.

Zun L. \& Gold I. (1986) A survey of the form of the mental status examination administered by emergency physicians. Annals of Emergency Medicine 15, 16-922. 